Revue internationale P.M.E.

Économie et gestion de la petite et moyenne entreprise

\title{
La criminalité contre les PME : étude exploratoire de victimisation et de prévention en Belgique francophone
}

\section{Bernard Surlemont, André Lemaître et Hélène Wacquier}

Volume 16, numéro 2, 2003

URI : https://id.erudit.org/iderudit/1008437ar

DOI : https://doi.org/10.7202/1008437ar

Aller au sommaire du numéro

Éditeur(s)

Presses de l’Université du Québec

ISSN

0776-5436 (imprimé)

1918-9699 (numérique)

Découvrir la revue

Citer cet article

Surlemont, B., Lemaître, A. \& Wacquier, H. (2003). La criminalité contre les PME : étude exploratoire de victimisation et de prévention en Belgique francophone. Revue internationale P.M.E., 16(2), 11-34.

https://doi.org/10.7202/1008437ar
Résumé de l'article

Cette étude exploratoire se penche sur la problématique de victimisation des PME. Basée sur l'organisation de tables rondes, la tenue d'entretiens en face à face et la réalisation d'une enquête auprès de 124 PME de Belgique francophone, la recherche dégage les types de victimisation et leur importance, les modes de prévention et l'approche de la gestion des conséquences d'un sinistre. Cette étude pluridisciplinaire réalisée par une équipe réunissant des spécialistes de la criminologie et de la gestion des PME investigue un terrain de recherche quasiment vierge. Elle souligne l'importance de distinguer les délits d'origine interne de ceux d'origine externe en raison des implications très différentes qu'ils ont au regard de la gestion. Les premiers relèvent en effet principalement de la gestion des ressources humaines tandis que les seconds relèvent plutôt de la gestion des risques. L'étude montre également que de nombreux délits ne sont pas rapportés aux services de police, ce qui autorise à penser que les statistiques policières sous-estiment largement l'étendue du phénomène de délinquance contre les entreprises. Elle révèle en outre que ces dernières ont une attitude plus réactive que proactive et que certains facteurs aggravent le risque de victimisation. Enfin, quelques pistes sont fournies en vue de la réalisation de recherches futures qui permettraient d'améliorer notre compréhension de ce phénomène longtemps resté dans l'ombre des projecteurs de la recherche.
Ce document est protégé par la loi sur le droit d'auteur. L'utilisation des services d'Érudit (y compris la reproduction) est assujettie à sa politique d'utilisation que vous pouvez consulter en ligne.

https://apropos.erudit.org/fr/usagers/politique-dutilisation/ 


\section{La criminalité contre les PME: étude exploratoire de victimisation et de prévention en Belgique francophone}

Bernard SURLEMONT

André LEMAÎTRE

Hélène WACQUIER

Université de Liège

\section{MOTS CLÉS}

\section{Délinquance - Criminalité - PME - Entreprise - Prévention Gestion de la délinquance - Cambriolage - Vol - Vandalisme}

\section{RÉSUMÉ}

Cette étude exploratoire se penche sur la problématique de victimisation des PME. Basée sur l'organisation de tables rondes, la tenue d'entretiens en face à face et la réalisation d'une enquête auprès de 124 PME de Belgique francophone, la

\section{LES AUTEURS}

Bernard Surlemont est diplômé de l'INSEAD (MBA et Ph.D.) et il a entrepris en 1995, après avoir œuvré 10 ans dans des entreprises internationales, une carrière académique comme professeur d'entrepreneuriat et directeur du Centre de recherche PME de l'Université de Liège (Belgique). Il est titulaire de la Chaire d'entrepreneuriat à l'Université de Lausanne depuis septembre 2000. Ses principaux domaines d'intérêt concernent la stratégie des entreprises en démarrage et en croissance. Adresse : HEC Lausanne, BFSH1, 1015 Lausanne, Suisse. Courriel : <Bernard.Surlemont@hec.unil.ch>.

ANDRÉ LEMAiTRE détient un doctorat en sciences criminologiques et enseigne à l'École de criminologie de l'Université de Liège. Ses principaux objets de recherche sont les politiques publiques de sécurité et la prévention de la criminalité. Adresse : Université de Liège, Faculté de droit, Service de criminologie, bd du Rectorat 3, B. 33, 4000 Liège, Belgique. Courriel : <alemaitre@ulg.ac.be>.

HÉLĖNE WACQUIER est diplômée de l'École d'administration des affaires de l'Université de Liège depuis 1993 et elle est chargée de recherche au Centre de recherche PME de l'Université de Liège. Dans ce cadre, elle a effectué des études et recherches dans les principaux domaines ayant trait à la gestion et aux facteurs qui favorisent le développement des PME et la création d'entreprises. 
recherche dégage les types de victimisation et leur importance, les modes de prévention et l'approche de la gestion des conséquences d'un sinistre. Cette étude pluridisciplinaire réalisée par une équipe réunissant des spécialistes de la criminologie et de la gestion des PME investigue un terrain de recherche quasiment vierge. Elle souligne l'importance de distinguer les délits d'origine interne de ceux d'origine externe en raison des implications très différentes qu'ils ont au regard de la gestion. Les premiers relèvent en effet principalement de la gestion des ressources humaines tandis que les seconds relèvent plutôt de la gestion des risques. L'étude montre également que de nombreux délits ne sont pas rapportés aux services de police, ce qui autorise à penser que les statistiques policières sous-estiment largement l'étendue du phénomène de délinquance contre les entreprises. Elle révèle en outre que ces dernières ont une attitude plus réactive que proactive et que certains facteurs aggravent le risque de victimisation. Enfin, quelques pistes sont fournies en vue de la réalisation de recherches futures qui permettraient d'améliorer notre compréhension de ce phénomène longtemps resté dans l'ombre des projecteurs de la recherche.

\begin{abstract}
This exploratory study focuses on the issue of SME victimisation. Based on round tables, interviews and a survey of 124 SME from the French-speaking part of Belgium, the research addresses the types of victimisation and their importance, the modes of prevention and the management of crime consequences. This multidisciplinary study, carried out by a team of criminology and SME management specialists, addresses an almost unexplored field of research. It underlines the importance of distinguishing crimes of internal origin from those of external origin because of their very different implications on key management. Internal crimes, indeed, mainly concern human resources management while external crimes are mostly related to risk management. The study also shows that a number of crimes are not reported to police, which suggests that police data considerably underestimate the importance of crime against businesses. Furthermore, it appears that SME adopt a more reactive than proactive attitude facing crime and also that some factors are likely to increase the risks of victimisation. Finally, some avenues are suggested for further research, which could possibly improve our comprehension of this issue.
\end{abstract}

\title{
RESUMEN
}

Este estudio exploratorio cubre la cuestión de la victimización de las PyMEs. Basándose en la organización de mesas redondas, de entrevistas en persona y en la realización de una encuesta cubriendo 124 PyMEs de la parte de habla francesa de Bélgica, nuestra investigación describe los diferentes tipos de victimización, así como su importancia, los modos de prevención y la gestión de las consecuencias de un siniestro. Este estudio pluridisciplinario, realizado por un equipo de especialistas en criminología y en gestión de las PyMEs, explora un campo de investigación prácticamente virgen. Se subraya la importancia de distinguir los delitos de origen interna y los delitos de origen externa, ya que ambos casos conllevan implicaciones muy distintas en lo que se refiere a la gestión. En el primer caso se trata esencialmente de gestión de los recursos humanos, mientras que el

Revue internationale P.M.E., vol. 16, $\mathrm{n}^{\mathrm{0}}$ 2, 2003

(C) 2003 - Presses de l'Université du Québec

Édifice Le Delta I, 2875, boul. Laurier, bureau 450, Sainte-Foy, Québec G1V 2M2 • Tél. : (418) 657-4399 - www.puq.uquebec.ca

Tiré de : Revue internationale P.M.E., vol. $16, \mathrm{n}^{\circ} 2$, sous la direction de Pierre-André Julien. 
segundo se refiere más bien a gestión de riesgos. Nuestro estudio destaca igualmente que un gran número de delitos no son señalados a las autoridades, lo cual sugiere que las cifras policiales subestiman fuertemente la magnitud del fenómeno de la delincuencia contra las empresas. Destacamos también el hecho de que la actitud de las empresas es más reactiva que proactiva, y de que algunos factores tienden a agravar el riesgo de victimización. En conclusión, sugerimos algunas pistas de investigación futura que permitirían mejorar aún más nuestra comprensión de este fenómeno, que ha permanecido mucho tiempo sin estudio.

\section{ZUSAMMENFASSUNG}

Diese Sondierungsstudie befasst sich mit der Opferrolle der KMU bei internen und externen Delikten. Aufgebaut auf der Organisation von Round tables und persönlichen Gesprächen wurde in Belgien eine Untersuchung bei 124 KMU aus dem französischen Sprachgebiet verwirklicht. Die Untersuchung setzte die Arten der Verschuldungsfrage und ihre Bedeutung, die Vorbeugungsmethoden und das Konzept der Behandlung eines Folgeschadens frei.

Diese bereichsübergreifende, interdisziplinäre Studie wurde von einem Team mit Spezialisten aus der Kriminologie und des KMU-Managements realisiert. Dabei entdeckte das Team ein fast jungfräuliches Forschungsgebiet. Die Studie unterstreicht die Bedeutung, dass zwischen Delikten mit internem Ursprung von solchen mit externen Ursprung zu unterscheiden ist. Die Unterschiede zeigen sich in ihren Auswirkungen und in der Art, wie die Vergehen behandelt werden. Delikte internen Ursprungs ergeben sich in der Tat hauptsächlich aus der Verwaltung der Humanressourcen, während sich die Externen eher aus der Verwaltung der Risiken ergeben.

Die Studie zeigt ebenfalls, dass zahlreiche Delikte nicht an die Polizei weitergeleitet werden. Somit unterschätzen sehr wahrscheinlich die Polizeistatistiken weitgehend die Bedeutung des Kriminalitätsphänomens gegen Unternehmen. Die Untersuchung zeigt ebenfalls, dass die Unternehmen mehr eine reaktive als eine proaktive Einstellung haben und somit bestimmte Faktoren aufweisen, die das Opferrisiko verschlimmern. Schließlich werden einige Spuren angesichts der Verwirklichung künftiger Forschungsarbeiten geliefert, die erlauben würden, noch unser Verständnis dieses Phänomens zu verbessern, das lang im Schatten der Projektoren der Forschung geblieben ist.

\section{Introduction}

Les individus ne sont pas les seuls à être victimes de la délinquance; l'entreprise peut également subir les dommages du vol ou de la dégradation de ses biens. Si l'on se réfère à certaines études nord-américaines, l'importance de ces dommages semble même considérable. Au Canada, des statistiques récentes évaluent le coût des vols par le personnel à plus de 22 milliards d'euros par an et estiment que le vol se répercute à raison de $15 \%$ dans le prix des biens et des services (Holt, 1993). Pour leur part, les statistiques du FBI estiment le coût de la criminalité dans les entreprises à près de 210 milliards d'euros par an (US Small Business Administration, 2000). Par ailleurs, la délinquance pose des problèmes de gestion

Revue internationale P.M.E., vol. 16, $\mathrm{n}^{\circ}$ 2, 2003

(C) 2003 - Presses de l'Université du Québec

Édifice Le Delta I, 2875, boul. Laurier, bureau 450, Sainte-Foy, Québec G1V 2M2 • Tél. : (418) 657-4399 - www.puq.uquebec.ca

Tiré de : Revue internationale P.M.E., vol. 16, n² , sous la direction de Pierre-André Julien. 
très complexes au sein des entreprises. Le premier domaine qui vient à l'esprit est évidemment celui de la gestion des risques : leur évaluation, leur prévention et la gestion des assurances de l'entreprise. Mais il convient aussi d'évoquer la gestion des ressources humaines, tant en termes de sensibilisation, de formation, de gestion psychologique que de droit du travail ou encore de politique du personnel. En effet, selon les cas, le personnel est tantôt victime, tantôt auteur d'actes de délinquance. Il peut également jouer un rôle dans l'établissement ou la gestion des sinistres. La problématique de la délinquance pèse aussi sur les systèmes de contrôle interne tant physiques (alarmes, contrôles d'accès, etc.) que financiers (mise en place des outils de gestion qui permettent de connaître et de mesurer l'ampleur des sinistres). Elle peut également avoir de fortes implications pour la gestion de la production lorsque des sabotages ou des vols de matériel peuvent considérablement perturber les planifications de production et influer sur les systèmes de contrôle de qualité. Des considérations liées à la délinquance peuvent en outre influencer la politique d'implantation de l'entreprise dans la mesure où certaines localisations apparaissent plus risquées que d'autres. Le commercial est, lui aussi, potentiellement concerné, la clientèle pouvant être elle-même soit victime, soit auteur de délits. Enfin, des actes tels que l'espionnage industriel ou l'incendie criminel menacent parfois la survie même de l'entreprise et c'est la stratégie de l'entreprise dans son ensemble qui est en jeu. Les implications sont donc multiples et importantes pour les entreprises en général et cruciales pour les PME en particulier. En effet, celles-ci ne sont pas a priori structurées pour mettre en œuvre une politique de détection et de prévention sophistiquée de la délinquance. Nos entretiens ont ainsi révélé que les plus petites entreprises ont une gestion très approximative de leurs stocks, rendant la détection de problèmes de vols de matières premières ou de produits finis très difficile. Par ailleurs, pour les PME, la délinquance peut avoir des conséquences fatales, car elles n'ont pas les ressources nécessaires pour supporter les pertes causées par certains actes délictueux. Ainsi, une étude du ministère du Commerce aux États-Unis évalue à $30 \%$ les faillites de PME causées par les frais résultant de la malhonnêteté de leurs employés (U.S. Department of Commerce, 1995).

Malgré l'importance du problème, il est surprenant de constater l'extrême rareté de la recherche consacrée à la victimisation des PME dans la littérature en gestion. Si l'on excepte les études statistiques qui tentent de mesurer l'impact de la victimisation d'entreprises (Walker, 1994, 1996), les rares études qui ont traité de ce sujet se sont attardées aux aspects de la gestion des ressources humaines ou à la prévention du vol en magasins (Felson et Clarke 1997; Gill, 1998). La seule étude qui a fait une première tentative pour cerner la problématique de la criminalité dans les PME est celle de Kuratko et al. (2000), qui investigue les méthodes de prévention dans les PME et évalue le degré de préoccupation que cette problématique suscite chez les dirigeants. Quant à la littérature francophone, les publications en la matière sont, à notre connaissance, tout simplement inexistantes. Cette 
pauvreté de la littérature portant sur un problème aussi important peut s'expliquer d'abord par ses implications interdisciplinaires au niveau de la gestion. En dehors du vaste cadre de la gestion des risques, il faut reconnaître que cette problématique ne constitue pas un champ d'investigation naturel des disciplines de base de la gestion. En outre, l'étude du phénomène de délinquance nécessite des rapprochements avec des domaines externes à la gestion dont, en particulier, celui de la criminologie. À cet égard, il est symptomatique de constater qu'en criminologie la majeure partie de ce qu'offre la littérature en matière de victimisation est basée sur des études concernant les individus et les ménages. De façon surprenante, très peu de recherches ont été menées sur les entreprises en tant que victimes de la criminalité. Abstraction faite de la tradition des études sur la délinquance économique et plus spécialement sur la fraude ${ }^{1}$, l'intérêt pour la recherche criminologique relative à la délinquance contre les entreprises est resté très limité. La première enquête de victimisation conduite aux Pays-Bas par Van Dijk, Van Soomeren and Partners BV (1990) avait été suivie en 1994 de l'International Crime against Businesses Survey ICBS (Van Dijk et Terlouw, 1996). Une autre source importante de connaissances sur la victimisation des entreprises est l'enquête australienne de 1993 (Walker, 1994, 1996) et le travail réalisé sur les parcs industriels par Johnston, Leitner, Shapland et Wiles en 1990 en Grande-Bretagne (Johnston et al., 1994). Notons également qu'en dehors de ces enquêtes, la plupart des études pouvant entrer dans notre champ d'intérêt concernent principalement les commerces (Felson et Clarke, 1997 ; Gill, 1998).

C'est la raison pour laquelle les Services de criminologie et le Centre de recherche PME et d'entrepreneuriat de l'Université de Liège ont combiné leurs efforts pour mener à bien une recherche exploratoire sur ce thème. L'objectif est de dresser un état des lieux des types de délinquance qui affectent le plus les PME ainsi que des stratégies que celles-ci mettent en œuvre pour gérer et prévenir cette délinquance. Notre intention était également de concentrer nos efforts sur un terrain de recherche totalement en friche, à savoir celui des PME principalement manufacturières. Précisons que nous avons exclu de notre champ d'analyse tant les fraudes dans les grandes entreprises que la délinquance dans les secteurs de la distribution et de la finance ; ces domaines ont fait l'objet d'études (Calavita, Pontell et Tillman, 1997 ; Punch, 1996) et concernent des problématiques très particulières que nous avons choisi d'exclure de notre champ d'investigation.

1. L'analyse quantitative de la fraude à caractère financier dont sont victimes les entreprises, tant des agents internes qu'externes, s'est progressivement affirmée comme un champ d'intérêt majeur de sociétés de consultance et d'associations d'experts en détection de la fraude, promouvant au passage leurs services. Ces enquêtes concernent la victimisation (KPMG, 1999), la perception du risque (Ernst et Young, 2000) et / ou les dispositifs préventifs et réactifs mis en place. Les données sont le plus souvent recueillies à l'échelon national, mais parfois intégrées à l'échelle régionale, voire mondiale.

Revue internationale P.M.E., vol. 16, $\mathrm{n}^{\circ}$ 2, 2003

(C) 2003 - Presses de l'Université du Québec 
Après une présentation du cadre méthodologique, nous passons en revue les résultats de notre analyse en abordant successivement les thèmes suivants : l'intensité de la délinquance en fonction des types, les mesures de prévention, l'identification des auteurs (interne ou externe) et de la victime (l'entreprise ou son personnel), la gestion du sinistre, l'évaluation des dommages et les adaptations postérieures aux sinistres. Nous analysons également l'impact de la localisation, du secteur et de la taille des entreprises sur les thèmes précisés ci-avant. Nous terminons par la présentation d'une typologie de situations de victimisation; celle-ci nous est apparue pertinente pour identifier et sérier les problématiques clés à gérer. Ce faisant, nous proposons une base permettant de mener d'autres recherches focalisées sur ces problématiques de gestion. Les conclusions mettent en évidence les différents dangers auxquels les entrepreneurs doivent faire face tant au regard de la prévention des sinistres que de la gestion de ses conséquences.

\section{Cadre méthodologique}

Un premier outil d'investigation a consisté dans le questionnaire d'une étude réalisée en Australie en 1993 sur la prévention de la délinquance dans les entreprises (Walker, 1994, 1996). Avec cette étude, l'objectif était d'établir certains parallélismes entre les cas australien et belge. Afin de tester ce premier outil et de lui apporter les aménagements nécessaires à son adéquation au contexte belge, 15 dirigeants d'entreprise de divers secteurs ont été invités à participer à une séancedébat. Grâce aux échanges d'expérience suscités par la lecture du document de travail, cette séance a notamment permis de dégager les principaux thèmes à investiguer et de concevoir un guide d'entretien plus concis et plus précis. Les rubriques suivantes y étaient abordées :

- profil des entreprises (activité, âge, appartenance éventuelle à un groupe, nombre de travailleurs, localisation);

- caractéristiques de l'environnement de l'entreprise (stationnement, bruit, ordures, présence non souhaitée d'enfants sur le site, éclairage public, degré de délinquance perçu, etc.);

- problématique de la délinquance (perception de l'intensité de la problématique et de son évolution);

- politique en matière de gestion et de prévention de la délinquance (budget sécurité, mesures de prévention mises en œuvre, participation à des actions interentreprises, contacts avec la police et les autorités locales, etc.);

- faits de délinquance subis au cours des cinq dernières années et, plus précisément, au cours des deux dernières années (vandalisme, incendies volontaires, cambriolages, fraudes, vol de [ou dans des] véhicules de la société, vols dans l'entreprise, agressions ou menaces, corruption, extorsion de fonds) :

Revue internationale P.M.E., vol. 16, $\mathrm{n}^{\circ}$ 2, 2003 
- nombre et nature des faits subis ;

- situation en matière d'assurances;

- coûts directs et indirects liés aux faits subis;

- auteurs des faits de délinquance;

- attitude en matière de plaintes auprès des autorités de police;

- mesures de sécurité supplémentaires mises en œuvre à la suite des faits subis.

L'étude de terrain a ensuite été réalisée selon deux approches complémentaires, soit par des entretiens en face à face et la mise en œuvre d'une enquête à plus large échelle, par voie téléphonique.

\subsection{La réalisation d'entretiens en face à face}

Ces entretiens ont été menés dans un but essentiellement exploratoire. Les dirigeants de 21 entreprises wallonnes ayant déjà collaboré avec l'Université de Liège dans le cadre d'autres recherches ont accepté de nous accorder un entretien d'une durée moyenne d'une heure.

\subsection{La réalisation d'une enquête à plus large échelle par voie téléphonique}

Une enquête à plus large échelle a été mise en œuvre pendant l'hiver 2000. Pour ce faire, en nous basant sur les premiers résultats de l'approche exploratoire, le guide d'entretien a une nouvelle fois été affiné et allégé, afin que le temps nécessaire pour l'administrer par voie téléphonique n'excède pas 20 minutes.

Ayant opté pour une enquête à caractère exploratoire, nous avons fixé à 100 le nombre minimal d'entreprises sur lesquelles fonder notre analyse. Selon un taux de réponse prévisionnel de $25 \%$, nous avons constitué un échantillon de 386 entreprises de la manière suivante : à partir de l'ensemble des PME wallonnes de 25 à 250 travailleurs créées avant le $1^{\text {er }}$ janvier 1999 et issues des industries manufacturières, du secteur de la construction, des transports terrestres, des activités informatiques et des services aux entreprises ${ }^{2}$, une sur trois en moyenne a été tirée aléatoirement de manière à respecter une représentativité d'au moins $40 \%$ par rapport à l'ensemble de la population des sous-secteurs ${ }^{3}$. Certains secteurs tels que le commerce de détail ou les banques et assurances ont volontairement été

2. La liste d'entreprises a été transmise par la Banque Nationale de Belgique

3. À l'exception du secteur de la construction pour lequel nous avons plafonné le nombre d'entreprises sélectionnées à 50 .

Revue internationale P.M.E., vol. 16, $\mathrm{n}^{\circ}$ 2, 2003

(C) 2003 - Presses de l'Université du Québec 
exclus, considérant qu'ils constituent une catégorie à part du point de vue de la délinquance ${ }^{4}$. Nous avons systématiquement contacté les entreprises sélectionnées afin de connaître le nom du responsable de production (ou du dirigeant dans le cas des entreprises de services) et de lui transmettre un courrier personnalisé exposant les objectifs de l'étude. En effet, il ressortait de la table ronde et des entretiens préliminaires que les PME confient généralement les tâches relatives à la sécurité et a fortiori à la gestion et à la prévention de la délinquance au responsable de production. Quelques jours après l'envoi du courrier, des enquêteurs ont joint les personnes ciblées par téléphone afin de leur donner de plus amples informations sur les objectifs de la recherche et de leur proposer de répondre au questionnaire «en direct» en les assurant du strict respect de leur anonymat. Étant donné le caractère sensible du sujet abordé, nous nous sommes engagés à tenir secrète l'identité des entreprises interrogées afin d'obtenir un maximum d'informations. Cet engagement nous a permis d'interroger directement les entreprises sur la plupart des thèmes liés à la délinquance. Il est toutefois apparu, au fil des entretiens préliminaires, que les thèmes de la corruption et de l'extorsion de fonds demeuraient très tabous. Afin de surmonter cette difficulté, nous avons orienté nos questions sur la perception de ces problématiques par les entreprises au sein de leur secteur d'activité plutôt que sur leur propre expérience en la matière. Cette technique, fréquemment utilisée en criminologie, nous a permis, de manière indirecte, de recueillir de précieuses informations sur le sujet.

En outre, pour tenter de maximiser le taux de réponse, nos services se sont engagés à transmettre une synthèse des résultats au terme de la recherche. Ajoutons que les enquêteurs avaient été formés à la problématique et longuement sensibilisés au questionnaire. Cette méthode a permis de réunir 124 questionnaires dûment remplis, soit un taux de réponse moyen de $32 \%$. Ces données ont principalement été analysées à l'aide de tables de fréquence et de contingence, la taille de notre échantillon ne permettant pas d'avoir recours à des analyses statistiques plus complexes.

4. De manière exhaustive, les secteurs exclus sont l'agriculture, la chasse, la sylviculture, la pêche, l'aquaculture, les industries extractives, la production et distribution d'électricité, de gaz, d'eau, le commerce et les réparations automobile et d'articles domestiques, l'HORECA, les activités financières, l'immobilier, les administrations publiques, l'éducation et la santé, les services collectifs, les services domestiques et les activités extra-territoriales. 


\section{Analyse des résultats}

\subsection{L'intensité de la délinquance}

L'étude de Walker a été retenue pour définir neuf catégories de délits dont l'entreprise pouvait être victime (Walker, 1994). Notre enquête révèle que près de la moitié des entreprises se déclarent affectées par des problèmes de délinquance et $15 \%$ d'entre elles considèrent même qu'il s'agit d'un grave problème pour leur entreprise. Le vol apparait comme l'un des délits les plus fréquents : plus de $70 \%$ des entreprises en ont été victimes au cours des cinq dernières années. Les cambriolages et vols de (ou dans des) véhicules personnels ou professionnels sur le lieu de travail ont concerné près d'une entreprise sur deux, la délinquance gratuite, plus d'une entreprise sur trois. En outre, $13 \%$ des entreprises ont été victimes d'actes de violence (agressions ou menaces à l'encontre du personnel) et une sur dix a été victime d'un incendie volontaire. Enfin, d'autres formes de délinquance telles que la corruption ou l'extorsion de fonds sont plus rares mais existent. Globalement, seules $6 \%$ des entreprises interrogées déclarent n'avoir connu aucun incident. Ajoutons que plus d'une entreprise sur cinq estime que les problèmes de délinquance l'affectant ont augmenté au cours des trois dernières années. Ces observations sont comparables à celles de Kuratko et al. (2000); dans cette recherche, les dirigeants estimaient que le problème s'aggravait dans $24 \%$ des cas. Dans la suite de cet article, nous nous concentrons sur les délits les plus fréquents, à savoir les vols, les cambriolages et les actes de vandalisme.

Il convient de noter que le degré de victimisation des entreprises influence sensiblement leur perception de la délinquance. Avec Kuratko et al. (2000), nous constatons que les entreprises les plus victimes sont aussi les plus nombreuses à considérer la délinquance comme une problématique sérieuse les affectant directement $(60 \%)$ et à la percevoir comme ayant augmenté au cours des trois dernières années $(40 \%)$.

TABleau 1

Fréquence de victimisation au cours des cinq dernières années

\begin{tabular}{lcrr}
\hline & $\begin{array}{c}\text { Jamais } \\
(\mathbf{\%})\end{array}$ & $\begin{array}{c}\text { Une fois } \\
(\mathbf{\%})\end{array}$ & $\begin{array}{c}\text { Plusieurs fois } \\
\mathbf{( \% )}\end{array}$ \\
\hline Délinquance «gratuite», vandalisme, etc. & 63,7 & 4,8 & 31,5 \\
Incendies volontaires & 89,5 & 8,1 & 2,4 \\
Cambriolages & 52,4 & 16,9 & 30,6 \\
Fraudes & 91,1 & 4,0 & 4,8 \\
Vols de (ou dans des) véhicules & 54,8 & 11,3 & 33,9 \\
Vols dans l'entreprise & 28,2 & 6,5 & 65,3 \\
Agressions ou menaces & 87,1 & 4,8 & 8,1 \\
Corruption & 98,4 & 0,8 & 0,8 \\
Extorsion de fonds & 98,4 & 1,6 & 0,0 \\
\hline
\end{tabular}

Revue internationale P.M.E., vol. 16, $\mathrm{n}^{\circ}$ 2, 2003 
Au niveau de l'analyse croisée, le tableau 2 montre que les entreprises les plus victimes de vandalisme, d'incendies volontaires, de cambriolages ainsi que de vols dans l'entreprise appartiennent aux secteurs de la métallurgie et de la construction. Plusieurs raisons peuvent être avancées pour expliquer ce constat. La métallurgie est une industrie traditionnelle en région wallonne, et les entreprises sont souvent logées dans des bâtiments anciens, vétustes et en dehors de tout ensemble d'entreprises (70\%). En outre, $90 \%$ d'entre elles sont localisées dans les régions traditionnellement spécialisées dans ce type d'industries lourdes: les provinces de Liège et du Hainaut qui, d'après nos résultats, connaissent les problèmes de délinquance les plus aigus. Par ailleurs, plus de $70 \%$ de ces entreprises ont plus de 100 travailleurs, ce qui constitue un facteur de risque supplémentaire (voir infra). En ce qui concerne le secteur de la construction, la délocalisation du travail sur chantier constitue la principale particularité. Les faits de délinquance les plus fréquemment subis sont d'ailleurs le vol dans les véhicules et la dégradation ou le vol de matériel sur le chantier. Notons que les secteurs de la R-D et des services aux entreprises apparaissent relativement plus touchés que la plupart des autres par la fraude et le secteur des transports, par les agressions. Il nous semble en outre intéressant de souligner les faibles pourcentages se rapportant aux délits tels que la corruption ou l'extorsion de fonds; à en croire les résultats, ces types de délits sont excessivement rares. Pourtant, lorsqu'on demande aux répondants s'ils pensent que la corruption constitue un problème en Belgique et en région wallonne, $85 \%$ répondent qu'il s'agit d'un problème important, voire très important, et près de $40 \%$ sont d'avis que ce genre de pratiques concernent leur secteur d'activité. Cette dissonance dans les résultats illustre à quel point ces types de délits sont tabous.

Si l'on observe les résultats par rapport à la taille, les entreprises de plus de 100 travailleurs apparaissent sensiblement plus victimisées : $42 \%$ d'entre elles contre $21 \%$ des entreprises plus petites déclarent avoir été victimes de plusieurs actes de vandalisme au cours des cinq dernières années. De même, $39 \%$ contre $23 \%$ déclarent avoir subi plusieurs cambriolages, $44 \%$ contre $24 \%$ plusieurs vols de ou dans des véhicules et $71 \%$ contre $60 \%$ plusieurs vols dans l'entreprise. En outre, $26 \%$ des entreprises de plus de 100 travailleurs, contre seulement $18 \%$ des autres, perçoivent les problèmes de délinquance les affectant comme ayant augmenté au cours des trois dernières années. Plusieurs hypothèses peuvent être avancées pour expliquer ces chiffres. Les grandes entreprises sont susceptibles de constituer des cibles de choix en raison de la tentation que leur visibilité et leur plus grande taille peuvent susciter. Leur taille peut également entraîner une plus grande déshumanisation de l'organisation poussant certains membres du personnel à céder plus facilement à la tentation de commetre des actes délictueux. Toutefois, nous ne pouvons exclure l'hypothèse que les chiffres des plus petites entreprises soient sous-estimés, celles-ci ayant une politique de prévention moins développée 


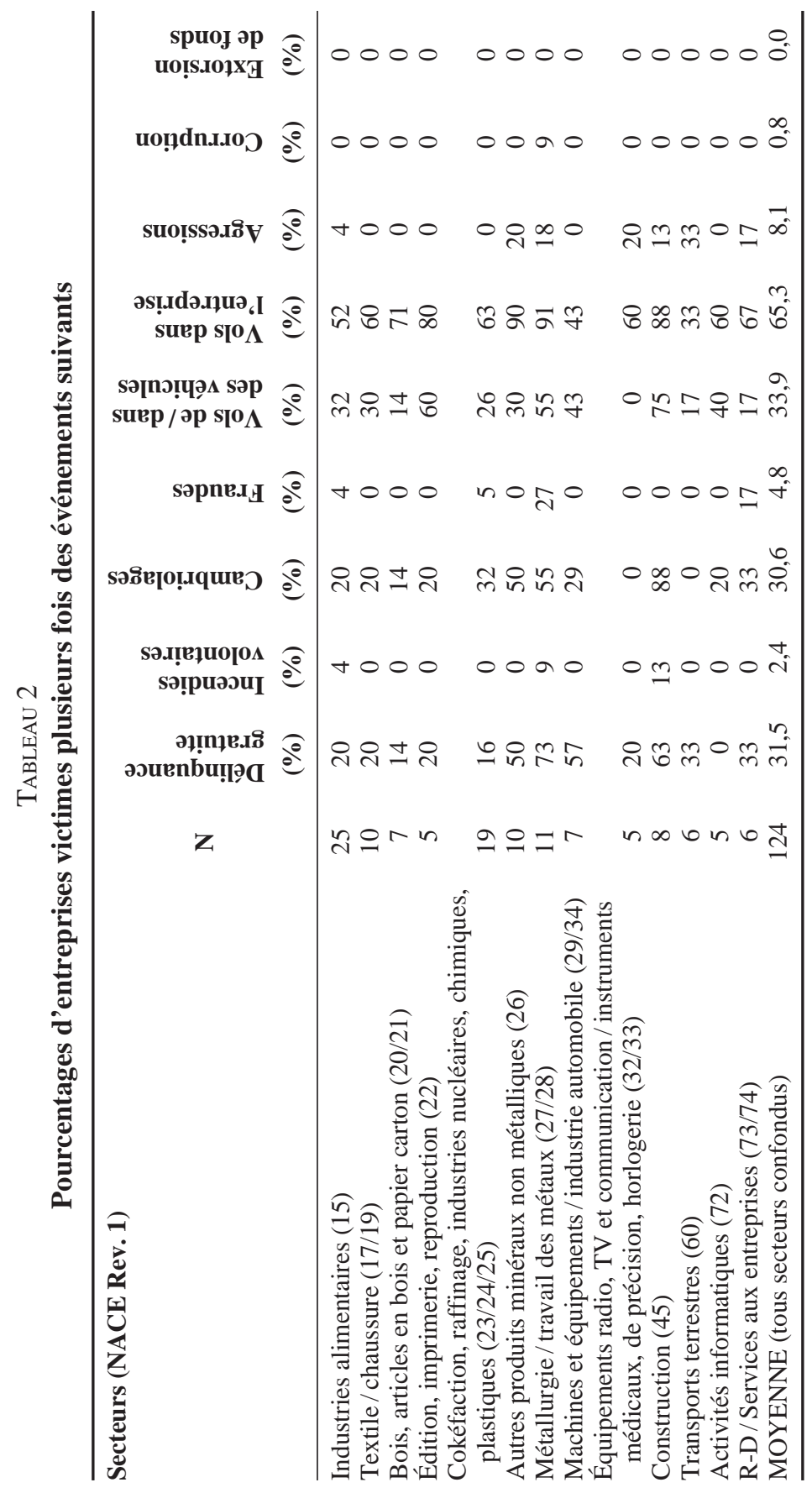

Revue internationale P.M.E., vol. 16, nº 2, 2003 
et, dès lors, étant moins bien armées pour établir les éventuels sinistres. Il est ainsi apparu que certaines petites entreprises n'exerçaient aucun contrôle systématique de leurs inventaires.

\subsection{Les mesures de prévention}

Devant l'ampleur du phénomène, il est surprenant de constater qu'en moyenne seules $20 \%$ des entreprises intègrent la problématique de la délinquance dans leur gestion. Moins de $15 \%$ ont attribué à une personne en particulier la responsabilité de la gestion des problèmes de délinquance et seules $5 \%$ ont un budget consacré à sa prévention. Cette observation doit toutefois être nuancée en fonction du secteur d'activité et de la taille des entreprises. Au regard des secteurs d'activité, les entreprises de services (activités informatiques et services aux entreprises) et celles ayant une activité intense de R-D sont plus de $50 \%$ à intégrer la problématique de la délinquance dans leur gestion, tandis que les PME relevant des industries manufacturières, de la construction et des transports sont généralement moins de $15 \%$ à intégrer cette problématique. Il semblerait donc que les entreprises où les conséquences d'actes de délinquance peuvent être stratégiques (p. ex., R-D et informatique) ont une plus nette tendance à intégrer cette dimension dans leur gestion. Les autres semblent plutôt considérer cela comme un mal nécessaire qui se «gère » de manière plus réactive que proactive à travers les polices d'assurance. Au regard de la taille des entreprises, on constate que les entreprises de plus de 100 travailleurs sont $27 \%$ à intégrer la problématique de la délinquance dans leur gestion et $10 \%$ à avoir un budget consacré à sa prévention contre respectivement $13 \%$ et $2 \%$ seulement des entreprises de moins de 100 travailleurs. Ce constat s'explique, d'une part, par le fait que ces plus grandes entreprises atteignent une masse critique qui leur permet une certaine structuration en matière de prévention et de gestion de la délinquance et, d'autre part, par le fait qu'elles sont plus victimisées que les plus petites. En effet, l'étude a mis en évidence le lien entre les actions de prévention et le niveau de victimisation; les entreprises les plus victimes sont plus nombreuses que la moyenne à intégrer la problématique de la prévention de la délinquance dans leur gestion. Il semblerait donc qu'il faille atteindre un seuil critique en la matière pour que des investissements soient consentis afin de pouvoir mieux la gérer ou la prévenir.

L'approche des mesures préventives varie fortement d'une entreprise à l'autre, mais consiste principalement en des mesures de «technoprévention» (alarmes antivol, vidéosurveillance, etc.). Sur ce plan, les attitudes n'apparaissent pas fondamentalement différentes selon que les entreprises aient été peu ou fortement victimisées. Toutefois, on observe un renforcement des mesures de prévention dans les cas de multivictimisations relatifs à certaines infractions comme le cambriolage ou le vol, confirmant, sur ce point précis, les résultats de Kuratko et al. 


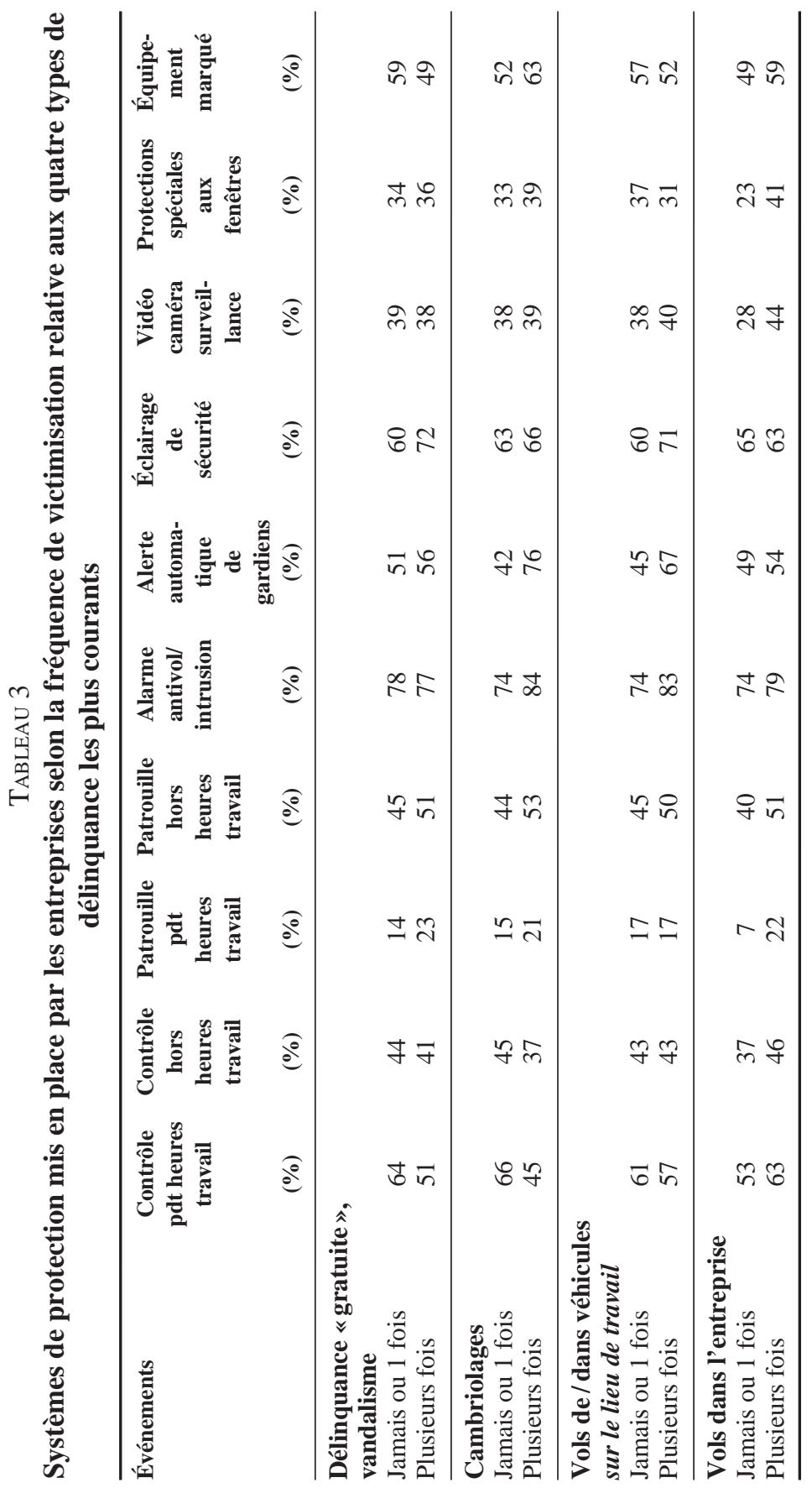

Revue internationale P.M.E., vol. 16, $\mathrm{n}^{\circ}$ 2, 2003 
(2000). Comme le montre le tableau 3, les mesures les plus significatives sont, dans le cas des cambriolages répétés, l'installation ou le renforcement des alarmes antivol, la mise en place d'alertes automatiques de gardiens, le marquage des équipements de valeur et le recours à des patrouilles en dehors des heures de travail. En ce qui concerne les vols répétés, les mesures les plus significatives sont le contrôle des entrées et sorties et le recours à des patrouilles pendant les heures de travail, la mise en œuvre de vidéosurveillance et le renforcement des protections aux fenêtres.

En ce qui a trait à la lutte concertée contre la délinquance, il existe relativement peu de coopération entre les entreprises. Moins d'une entreprise sur cinq a été sollicitée pour prendre part à des actions communes organisées dans sa zone. Parmi ces entreprises, une sur deux a effectivement pris part aux actions organisées et les a généralement jugées efficaces ( $65 \%$ de satisfaits). Enfin, en matière d'assurances, l'attitude varie fortement suivant le dommage. Les entreprises sont, la plupart du temps, assurées contre l'incendie criminel (bien que $10 \%$ d'entre elles déclarent ne pas avoir encore d'assurances contre ce sinistre). Près de la moitié sont assurées contre les cambriolages et les vols de ou dans les véhicules. En revanche, elles ne sont qu'un peu plus de $26 \%$ à s'assurer contre le vol dans l'entreprise. Cette dernière observation pourrait s'expliquer par la difficulté à démontrer et à gérer les conséquences des vols internes aux yeux des assurances dans la mesure où ces vols sont, la plupart du temps, le fait des membres du personnel de l'entreprise. Il convient aussi de relever que, au cours des entretiens, nombre d'entreprises donnaient l'impression de ne pas savoir exactement contre quels risques elles étaient assurées, laissant croire à une gestion très approximative en la matière.

\subsection{Les auteurs de la délinquance}

Il ressort de notre analyse que les faits de délinquance, lorsqu'ils sont établis, peuvent avoir pour auteurs des personnes tant de l'interne que de l'externe de l'entreprise. À cet égard, nous ne disposons pas d'informations permettant de conclure qu'un type de délinquance est dominant par rapport à un autre. Toutefois, la problématique est très différente selon ces deux cas de figure.

La délinquance interne relève avant tout de la gestion des ressources humaines. Parmi les entreprises interrogées, 43,5\% considèrent ce type de délinquance comme le plus facile à gérer en raison des moyens de pression dont l'entreprise dispose à l'égard du personnel lorsque, toutefois, les auteurs des délits sont formellement identifiés. Par ailleurs, elles sont 17,7\% à penser exactement le contraire: ces situations sont les plus difficiles à gérer parce qu'elles amènent immanquablement un déséquilibre, une perte de confiance et un climat de suspicion au sein de l'entreprise. 
Le vol, qui a touché près des deux tiers de notre échantillon au cours des deux dernières années, est au sommet du hit-parade des faits de délinquance de source interne. Nos entretiens en face à face ont révélé à cet égard une assimilation très subjective de la notion de « vol » en tant que délit. La frontière entre le tolérable et l'intolérable peut varier considérablement d'une entreprise à l'autre ou d'un secteur à l'autre. L'usage des moyens de l'entreprise à des fins personnelles est considéré par certains dirigeants comme du vol pur et simple, mais par d'autres comme un avantage en nature accordé tacitement au personnel. Le niveau de tolérance à ce type de comportement semble d'ailleurs lié à la rareté ou au caractère «irremplaçable» de la main-d'œuvre. Pour illustrer ce constat, un dirigeant d'entreprises a déclaré au cours d'un entretien qu'il ne condamnerait pas le « vol » de petit matériel de bureau par ses informaticiens, ceux-ci étant actifs dans un secteur à forte valeur ajoutée. Par contre, dans une autre de ses entreprises, soumise à des marges beaucoup plus restreintes, il réprimanderait immédiatement les ouvriers qui tenteraient de subtiliser des outils de travail. Enfin, de manière plus générale, soulignons que la notion de délinquance, dans le chef des dirigeants de PME, est étroitement liée à celle de faute grave. Un acte est considéré comme un fait de délinquance s'il apparaît justifié aux yeux du chef d'entreprise de licencier son auteur pour faute grave.

Les biens les plus visés par le vol sont sans conteste l'outillage et le matériel de travail (dans $60 \%$ des cas) ainsi que l'argent et les portefeuilles (37\%). Sont également visés, dans une moindre mesure, les objets appartenant au personnel et les biens mis à la disposition du personnel par l'entreprise (p. ex., les vêtements de travail), le matériel informatique et électronique ainsi que les matières premières ou les produits finis.

La délinquance externe relève d'abord d'un problème de protection et de gestion d'assurances. Les PME sont seulement 3,2\% à considérer qu'il s'agit du type de délinquance le plus difficile à gérer, principalement en raison de la fréquence des délits dont elles sont victimes (il s'agit dans ces cas d'entreprises littéralement harcelées). Ces résultats confirment nos entretiens exploratoires qui démontraient que la délinquance externe se gère de manière très rationnelle et quasi systématique et ne pose guère de problèmes majeurs à l'entreprise sauf lorsque des membres de celle-ci sont personnellement victimes des délits.

Les faits de délinquance externe les plus fréquents sont le vandalisme ( $31 \%$ de victimes au cours des deux dernières années), le cambriolage (29\%) et le vol de ou dans les voitures ( $28 \%$ ). Les actes de vandalisme les plus fréquents sont le bris de vitre $(29 \%)$, les tags sur les murs de l'entreprise (29\%), les graffitis à l'intérieur de l'entreprise (21\%), la dégradation de véhicules (21\%) et la dégradation de gros matériel ou de matériel de chantier $(18 \%)$.

Revue internationale P.M.E., vol. 16, $n^{\circ}$ 2, 2003

(C) 2003 - Presses de l'Université du Québec 


\subsection{La gestion du sinistre}

La gestion du sinistre, outre l'identification de son auteur, consiste en l'évaluation et la réparation des dégâts ainsi que les déclarations aux assurances et à la police. C'est ce dernier point que nous approfondissons ci-après.

Le fait que l'entreprise dépose ou non une plainte est tributaire d'une série de facteurs dont, notamment, le type d'infraction subie, le fait que l'auteur des actes de délinquance soit externe ou interne à l'entreprise et le fait que les préjudices portent atteinte à des biens de l'entreprise ou à des biens personnels. Dans ce dernier cas, la charge de la plainte est la plupart du temps reportée sur l'individu directement concerné. L'enquête révèle que les entreprises ne portent plainte que dans $20 \%$ des cas d'agression, $29 \%$ des cas de fraude, $32 \%$ des cas de vol, $50 \%$ de faits de vandalisme, $57 \%$ des atteintes aux véhicules et $80 \%$ des cambriolages. Il arrive même que des incendies volontaires ne soient pas déclarés. Toutefois, le fait qu'un incident ne fasse pas l'objet d'une dénonciation ne signifie pas qu'il ne connaîtra aucune suite. Un certain nombre de problèmes sont réglés «en famille» et, en particulier, tout ce qui touche à la délinquance interne, tant pour des raisons de politique de personnel que pour ne pas ternir l'image de l'entreprise. Ainsi, au cours des deux dernières années, plus des deux tiers des entreprises n'ont rapporté aucun vol auprès des services de police; la grande majorité estiment que les faits sont généralement mineurs et que déposer une plainte constituerait une perte de temps. En outre, la grande majorité des entreprises n'ont aucune certitude quant à l'identité des auteurs des vols ; même lorsque les présomptions sont fortes (celles-ci portant sur des personnes internes à l'entreprise dans $91 \%$ des cas), elles ne disposent généralement pas de preuves suffisantes pour espérer faire aboutir une plainte.

En matière de cambriolage, le comportement diffère notablement, car, bien que dans plus de $80 \%$ des cas, les entreprises déclarent n'avoir aucune idée de l'identité de l'auteur des faits, près des trois quarts d'entre elles ont porté plainte auprès de la police à la suite de tels faits. C'est un délit qui a donc largement plus d'écho que le vol interne. Ce comportement particulier à l'égard des services de police est lié au fait que les compagnies d'assurances conditionnent souvent leur intervention à l'existence d'une déposition à la police. Avec les incendies, le cambriolage constitue l'un des risques contre lesquels les entreprises sont le plus systématiquement couvertes.

D’une manière générale, ces informations de terrain montrent que la délinquance contre les entreprises est beaucoup plus répandue que ne pourraient le laisser croire les statistiques policières. En effet, les délits sont loin d'être systématiquement rapportés aux services de police, sans compter qu'ils se produisent parfois à l'insu de l'entreprise elle-même. Enfin, en ce qui concerne l'action des services de police

et de la gendarmerie en matière de gestion et de prévention de la délinquance, on

Revue internationale P.M.E., vol. 16, $\mathrm{n}^{\circ}$ 2, 2003

(C) 2003 - Presses de l'Université du Québec 
constate que la satisfaction et la confiance des entreprises s'érodent en fonction de l'importance de la victimisation dont celles-ci ont souffert. Entre les entreprises les moins victimes et les entreprises les plus affectées, l'indice de satisfaction enregistre une chute de $66 \%$ à $46 \%$. Dans ce cas, ce n'est pas tant la compétence policière qui est remise en cause que l'absence de suite donnée et surtout le peu d'information communiquée sur l'évolution des enquêtes une fois la plainte déposée.

\subsection{L'évaluation des dommages}

Les entreprises disposent généralement de peu d'informations sur le coût réel de la délinquance. D'une part, elles ne possèdent pas d'outils analytiques leur permettant d'apprécier l'importance des dommages et, d'autre part, le pragmatisme des dirigeants de PME les amène à juger ce type d'estimation inutile à partir du moment où les dommages ont effectivement été subis. C'est au chapitre du cambriolage que les entreprises victimes déclarent le plus fréquemment avoir une connaissance précise du coût direct (plus de $80 \%$ ) ; cette situation est liée à l'exigence des compagnies d'assurances de disposer d'une évaluation du sinistre, voire d'une expertise pour indemniser l'entreprise. Pour les entreprises victimes, le dommage moyen s'est élevé, pour les deux dernières années, à $€ 17.350$. Les effets indirects les plus souvent soulignés sont les retards dans la production ou la prestation des services (17\%) et le chômage technique du personnel (14\%). Pour tous les autres délits, moins de la moitié des entreprises victimes déclarent connaître le coût du dommage qu'elle a subi. Lorsqu'il est connu, ce dernier s'élève généralement à moins de $€ 12.400$ en moyenne par entreprise pour les deux dernières années. En dehors de la perte financière proprement dite, près d'un quart des vols et $10 \%$ des actes de vandalisme ont des conséquences indirectes sur la production.

\subsection{Les adaptations postérieures aux sinistres}

Les informations recueillies permettent de conclure que les PME ont une approche plutôt réactive que proactive en matière de sécurité. C'est à nouveau avec le cambriolage que les entreprises sont les plus réactives. Cinq entreprises victimes sur six ont adopté des mesures de sécurité supplémentaires. Les deux plus fréquentes sont l'installation d'alarmes antivol et le renforcement des protections aux portes et aux fenêtres (un tiers des entreprises). D'autres mesures fréquentes sont le recours à des services de gardiennage, la clôture du site et un stockage plus sécurisé du matériel et des objets de valeur (respectivement 19\%, $14 \%$ et $11 \%$ ). Pour le vandalisme, seule une PME victime sur deux a pris des mesures de protection supplémentaires. Parmi celles-ci, les plus fréquentes sont le recours à des entreprises de gardiennage (16\%), la mise en place de systèmes de contrôle d'accès $(13 \%)$ ou encore le renfort des protections aux portes et fenêtres et l'installation de caméras de surveillance (respectivement $10 \%$ et $5 \%$ ). Au regard des vols, les

Revue internationale P.M.E., vol. 16, $\mathrm{n}^{\circ}$ 2, 2003

(C) 2003 - Presses de l'Université du Québec 
réactions sont à la fois techniques (p. ex., renforcement des serrures dans $25 \%$ des cas) et humaines, par le biais d'actions de formation et de sensibilisation (16\% des cas).

\subsection{Les facteurs aggravants}

Nous avons étudié de plus près les entreprises les plus victimes afin de relever d'éventuels facteurs aggravants.

Pour réaliser cette analyse, nous avons établi trois catégories d'entreprises selon le degré de délinquance qui les a affectées au cours des cinq dernières années. Le groupe A est constitué des entreprises les moins affectées ${ }^{5}$, le groupe C, des plus affectées par tous les types de délinquance. Le tableau 4 présente, au sein de chacun des groupes $\mathrm{A}, \mathrm{B}$ et $\mathrm{C}$, pour chaque fait de délinquance étudié, le pourcentage d'entreprises qui ont été victimes plus d'une fois du délit en question.

TABLEAU 4

Regroupement des entreprises selon le degré de victimisation

Pourcentage d'entreprises victimes plusieurs fois des faits de délinquance suivants

\begin{tabular}{lccc}
\hline & $\begin{array}{c}\text { Groupe A } \\
\mathbf{N = 4 7} \\
(\boldsymbol{\%})\end{array}$ & $\begin{array}{c}\text { Groupe B } \\
\mathbf{N = 3 8} \\
(\boldsymbol{\%})\end{array}$ & $\begin{array}{c}\text { Groupe C } \\
\mathbf{N}=\mathbf{3 9} \\
(\boldsymbol{\%})\end{array}$ \\
\hline Délinquance «gratuite», vandalisme, etc. & 11 & 11 & 77 \\
Incendies volontaires & 0 & 3 & 5 \\
Cambriolages & 6 & 24 & 67 \\
Fraudes & 0 & 0 & 15 \\
Vols de (ou dans des) véhicules & 6 & 34 & 67 \\
Vols dans l'entreprise & 32 & 82 & 90 \\
Agressions ou menaces & 2 & 11 & 13 \\
Corruption & 0 & 0 & 3 \\
Extorsion de fonds & 0 & 0 & 0 \\
\hline
\end{tabular}

À partir de ces groupes d'entreprises, nous avons ensuite dégagé les caractéristiques qui permettent de les discriminer au mieux afin de mettre en relief d'éventuels «facteurs de risque ». Ainsi, nous avons observé un niveau plus élevé

5. Pour ce qui concerne la répartition par groupe, nous avons procédé comme suit: pour chacun des neuf types de délinquance étudiés dans l'enquête, nous avons demandé aux entreprises si elles en avaient été victimes jamais (1), une fois (2) ou plusieurs fois (3) au cours des cinq dernières années. Pour chaque entreprise, nous avons ensuite additionné les cotes obtenues pour les neuf types de délinquance, la somme pouvant s'étaler de 9 (minimum : jamais victime d'aucun délit) à 27 (plusieurs fois victime de tous les délits). Le groupe A est constitué des entreprises dont la cote est comprise entre 9 et 11 , le groupe B entre 12 et 14 et le groupe C, plus de 14 . 
de délinquance gratuite et de vandalisme en milieu urbain qu'en milieu rural (respectivement $50 \%$ contre $22 \%$ d'entreprises plusieurs fois victimes au cours de trois dernières années). Ce critère de localisation en ville ou à la campagne n'apparaît pas comme discriminant au regard des autres catégories de délinquance. Par ailleurs, la localisation dans un parc industriel versus en dehors de tout ensemble d'entreprises semble, à première vue, déterminante. Contrairement à ce qu'avait observé l'étude australienne de 1993 (Walker, 1996), les entreprises localisées dans des parcs industriels, commerciaux ou autres apparaissent sensiblement moins affectées que celles situées en dehors de tout ensemble d'entreprises. En effet, $68 \%$ des entreprises les moins victimes (A) sont situées dans un ensemble d'entreprises, tandis que $82 \%$ des entreprises les plus victimes (C) sont situées en dehors de tout ensemble d'entreprises. Le fait que la constatation s'applique à tous les types de délinquance, y compris le vol « interne » pour lequel, manifestement, la localisation ne devrait pas avoir d'influence, suggère que la distinction parc industriel/hors parc industriel n'est en réalité pas pertinente en elle-même, mais dissimule d'autres facteurs de risque. Parmi ceux-ci, le secteur d'activité semble jouer un rôle prépondérant. Deux secteurs apparaissent nettement plus affectés par tous les types de délinquance que les autres; il s'agit des secteurs de la construction ainsi que de la métallurgie et du travail des métaux dont respectivement $88 \%$ et $82 \%$ des entreprises sont «multivictimisées» (C). Les taux de victimisation de ces deux secteurs sont extrêmement élevés pour tous les types de délinquance. Le secteur de la construction apparait plus sensible que tout autre aux cambriolages, aux vols dans les véhicules, ainsi qu'aux incendies volontaires. Cette observation est probablement due au fait que les chantiers sont moins bien protégés et plus facilement accessibles au public que les locaux d'une entreprise. La métallurgie enregistre des taux plus élevés que la moyenne en matière de vols internes et d'agressions et de menaces. Dans la mesure où, respectivement $88 \%$ et $73 \%$ des entreprises de la construction et de la métallurgie sont situées en dehors de tout ensemble d'entreprises, il appert que le critère «secteur d'activité » pourrait être prépondérant par rapport au critère de localisation «parc industriel / hors parc industriel ». Une étude complémentaire est cependant nécessaire pour mesurer de manière plus précise l'importance relative de ce critère.

En dehors du secteur d'activité proprement dit, l'enquête montre, en toute logique, que les entreprises qui exercent une activité de stockage ou de vente sont relativement plus touchées que celles qui se cantonnent à une fonction de production ou de prestation de services. Elles sont bien sûr surtout plus affectées sur le plan des vols et cambriolages.

En outre, soulignons que le fait d'avoir recours systématiquement ou régulièrement à de la main-d'œuvre intérimaire n'apparaît pas comme un critère d'accroissement du niveau de délinquance dans les entreprises. 
Il est par ailleurs surprenant de constater que les entreprises du groupe $\mathrm{C}$ ne sont pas mieux assurées que celles qui sont moins affectées. Les entreprises les plus victimes sont également celles qui sont les plus nombreuses à considérer la délinquance comme un sérieux, voire un très grave problème. Elles sont également les plus nombreuses à déclarer que les problèmes de délinquance les affectant ont augmenté au cours des trois dernières années. La problématique de la gestion de la délinquance fait partie intégrante de la politique de gestion de l'entreprise dans $26 \%$ des entreprises du groupe $\mathrm{C}$ contre $11 \%$ des entreprises du groupe $\mathrm{A}$, se traduisant, notamment, par l'attribution de la responsabilité de la gestion des problèmes de délinquance à une personne en particulier. Cependant, malgré leur situation de «victimisations répétées », seules $8 \%$ des entreprises $\mathrm{C}$ ont un budget consacré à la prévention des problèmes de délinquance (contre $2 \%$ des entreprises A). En matière de mesures de prévention, le tableau 5 ci-après offre une comparaison entre le groupe $\mathrm{A}$ et le groupe $\mathrm{C}$.

TABLeau 5

\section{Comparaison des systèmes de prévention en fonction des groupes d'entreprises}

\begin{tabular}{|c|c|c|}
\hline & $\mathbf{A}$ & $\mathbf{C}$ \\
\hline Système de contrôle des entrées et sorties & $62 \%$ & $54 \%$ \\
\hline Système de contrôle des entrées et sorties & & \\
\hline en dehors des heures de travail & $36 \%$ & $38 \%$ \\
\hline $\begin{array}{l}\text { Gardiennage ou patrouilles de sécurité sur le site } \\
\text { pendant les heures de travail }\end{array}$ & $11 \%$ & $23 \%$ \\
\hline $\begin{array}{l}\text { Gardiennage ou patrouilles de sécurité sur le site } \\
\text { en dehors des heures de travail }\end{array}$ & $38 \%$ & $54 \%$ \\
\hline Système d'alarme antivol, anti-intrusion & $79 \%$ & $74 \%$ \\
\hline $\begin{array}{l}\text { Système d'intervention des gardiens de la sécurité } \\
\text { en cas d'alarme }\end{array}$ & $45 \%$ & $62 \%$ \\
\hline Éclairage spécial de sécurité & $60 \%$ & $72 \%$ \\
\hline Vidéo ou caméra de surveillance & $30 \%$ & $49 \%$ \\
\hline $\begin{array}{l}\text { Protections spéciales aux fenêtres } \\
\text { (vitres, barreaux, verrous, grillage, etc.) }\end{array}$ & $32 \%$ & $46 \%$ \\
\hline $\begin{array}{l}\text { Équipements de valeur marqués d'un numéro de série } \\
\text { ou d'identification }\end{array}$ & $53 \%$ & $54 \%$ \\
\hline
\end{tabular}

Les différences touchent principalement le gardiennage pendant et en dehors des heures de travail, l'alerte automatique de gardiens, l'éclairage de sécurité, la vidéosurveillance et les protections renforcées aux fenêtres. Les entreprises situées dans les parcs industriels sont plus nombreuses à prendre des mesures de prévention que les entreprises localisées en dehors de tout ensemble. À l'égard de l'action des services de police et de gendarmerie, les entreprises $\mathrm{C}$ sont plus de $50 \%$ à être 
insatisfaites contre $19 \%$ seulement des entreprises A. De même, $41 \%$ des entreprises $\mathrm{C}$ sont insatisfaites de l'action des autorités locales en matière de délinquance contre seulement $9 \%$ des entreprises A.

\section{Conclusion}

Cette étude exploratoire a mesuré l'importance du phénomène de victimisation d'entreprises, largement sous-estimé par les statistiques policières. Elle a également montré que les aspects de la gestion d'entreprise liés à cette problématique étaient souvent sous-estimés, voire ignorés. Il semble falloir être affecté par la délinquance pour tenter de la prévenir. Les comportements et réactions varient également en fonction des délits.

Par ailleurs, la nature des problématiques varie considérablement selon que l'auteur des faits est interne ou externe à l'entreprise. La figure 1 synthétise les principales conclusions dégagées à ce sujet par notre analyse. On y distingue, d'une part, l'auteur des faits de délinquance (interne-externe) et, d'autre part, l'identité de la victime (l'entreprise ou les membres de son personnel).

FIGURE 1

Typologie des situations de victimisation d'entreprises

\begin{tabular}{|c|c|c|c|}
\hline & \multicolumn{2}{|c|}{ ORIGINE DU DÉLIT } \\
\hline & & Interne & Externe \\
\hline \multirow{2}{*}{ 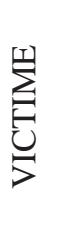 } & Entreprise & $\begin{array}{c}\text { GRH } \\
\operatorname{Cas~n}^{\circ} 1\end{array}$ & $\begin{array}{c}\text { GESTION DES RISQUES } \\
\operatorname{Cas~n~}^{\circ} 3 \\
\end{array}$ \\
\hline & Personnel & $\begin{array}{c}\text { GRH / INFORMATION } \\
\operatorname{Cas~n}^{\circ} 2\end{array}$ & $\begin{array}{l}\text { INFORMATION / GRH } \\
\qquad \operatorname{Cas~n~}^{\circ} 4\end{array}$ \\
\hline
\end{tabular}

Le premier type de situation vise principalement le vol par le personnel d'outils et de petits matériels appartenant à l'entreprise. Dans ce cas, bien que l'on soit certain de l'origine interne des délits, l'auteur même de ceux-ci n'est pas toujours formellement identifié. Cette situation est généralement considérée comme la plus difficile à gérer. En effet, elle engendre suspicion, perte de confiance et peut, dans certains cas, perturber très fortement l'organisation de l'entreprise. Dans tous les cas, la situation $\mathrm{n}^{\circ} 1$ relève essentiellement de la gestion des ressources humaines. Les entreprises ont généralement tendance à traiter ce genre de problèmes «en famille» et à ne pas déclarer ces faits aux services de police. Il appert que la notion de délit dans cette catégorie varie fortement en fonction du profil de 
travailleur, du secteur de l'entreprise et de la sensibilité de ses dirigeants. Ces types de délits peuvent être prévenus par la sensibilisation, une responsabilisation accrue ou le renforcement de la formation du personnel et la mise en place de systèmes de contrôle renforcés pouvant aller jusqu'à la fouille au sortir des ateliers (accès contrôlés, systèmes d'alarme, vidéosurveillance ou patrouilles pendant les heures de travail). Le cas $\mathrm{n}^{\circ} 2$ occasionne moins de préjudices directs à l'entreprise, mais il pose de sérieux problèmes de confiance et d'ambiance au sein du personnel. Il vise principalement des situations de vols de portefeuilles, de sacs ou dans les voitures. Les mesures de prévention se traduisent par la sensibilisation et la formation du personnel sur les précautions à prendre sur les lieux de travail, voire par la sécurisation des lieux de parcage et de dépôt d'effets personnels. Les cas no 3 et n ${ }^{\circ} 4$ relèvent plus de la criminalité «professionnelle » et de la délinquance externe. En ce qui concerne le cas $n^{\circ} 3$, il s'agit principalement de cambriolages et d'actes de vandalisme. Ces derniers constituent généralement des situations moins délicates à gérer dans la mesure où il s'agit surtout de problématiques de protection (technoprévention) et de couverture par les assurances. Les premiers entretiens avaient déjà mis en évidence que, même si ces faits peuvent gêner le fonctionnement optimal de l'entreprise, ils relèvent toutefois d'une gestion rationnelle au travers des polices d'assurance et, à ce titre, sont habituellement considérés comme moins problématiques par les PME. Il s'agit clairement d'une approche qui relève davantage de la gestion des risques. Quant aux cas $n^{\circ} 4$, ils concernent principalement le vol de ou dans les voitures du personnel. Dans la plupart des cas, l'entreprise se considère peu concernée par ces situations; il appartient à la victime d'entreprendre les démarches pour donner suite au délit (identification de l'auteur, déclaration à la police et déclaration de sinistre). Même si ce type de problèmes peut susciter un sentiment d'insécurité et, in fine, démotiver le personnel, les principales actions préventives prises par les entreprises à cet égard visent l'information du personnel. Certaines proposent toutefois des mesures plus concrètes telles que la sécurisation des stationnements réservés au personnel.

Cette recherche constitue une première analyse d'un phénomène souvent laissé dans l'ombre. À cet égard, elle a mis en évidence plusieurs champs d'intervention, tant sur un plan empirique que sur celui des implications pratiques, spécifiques à des secteurs généralement réputés moins victimes que des secteurs tels que les banques ou les commerces. Ces champs d'intervention concernent notamment la gestion du personnel et la formation, la gestion des risques et les assurances et la gestion budgétaire et le contrôle interne. Vu l'extrême diversité du phénomène, nos investigations nous amènent également à recommander de cibler des recherches futures sur des champs plus spécifiques. Trois niveaux d'analyse possibles se dégagent. Le premier porte sur l'identification du sinistre. Sans pouvoir le mesurer de manière précise, notre étude a mis le doigt sur un phénomène très important, celui de la non-détection des sinistres. En effet, la 
«non-prévention » entraîne, dans de nombreux cas, une absence d'identification du sinistre conduisant à une sous-estimation des dommages pouvant aller jusqu'à la mise en danger de l'existence même des entreprises, en particulier des plus petites d'entre elles. Ainsi, au cours des entretiens préliminaires, certains dirigeants ont révélé avoir cessé certaines activités non rentables, se rendant compte, a posteriori, que ce manque de rentabilité provenait d'un «coulage» des stocks. À cet égard, il conviendrait de définir et d'analyser les outils susceptibles de permettre aux PME de détecter les sinistres. Parmi ceux-ci, les systèmes de contrôle comptables internes viennent rapidement à l'esprit, mais les PME gagneraient également à développer une politique plus proactive en matière de prévention, qui pourrait notamment se traduire par une identification systématique des risques et de leurs modes de prévention, une formation adéquate du personnel, un contrôle et une surveillance des accès. Eu égard à l'absence totale de recherches en la matière, tous ces thèmes constituent autant de pistes de recherches futures.

Le deuxième niveau d'analyse porteur d'enseignements, considérant que le sinistre est détecté, vise la détection de l'origine du délit, interne ou externe. En effet, la figure 1 laisse dans l'ombre une question essentielle qu'est l'identification formelle de l'auteur des actes de délinquance. Nos entretiens ont révélé qu'il s'agit d'une question cruciale qui est trop souvent ignorée. L'inconnue portant sur l'origine du sinistre laisse souvent la PME démunie par rapport à la suite qu'il convient de lui donner, p. ex., déclarer le sinistre aux services de police ou mener une enquête à l'interne. Cette décision est souvent délicate à prendre. Faire aveu de doute risque, par exemple, de détruire la confiance au sein du personnel. De même, agir à l'égard de membres du personnel sans certitude quant à l'origine interne du délit est très déstabilisant. Force est de reconnaître qu'à ce jour aucune recherche n'a mis en évidence la moindre piste sur les comportements et réactions qu'il y a lieu d'adopter pour gérer ce type de situation.

Enfin, le troisième niveau d' analyse intervient lorsque le sinistre et son origine sont clairement établis. Elle vise alors à l'identification précise de l'auteur des faits. Lorsque l'origine est clairement externe, l'entreprise se tournera vers ses assurances et les services de police (ne serait-ce que pour officialiser l'existence d'un sinistre à l'égard des compagnies d'assurances); la gestion du sinistre est alors avant tout «technique». Lorsque l'origine est interne, la gestion du problème relèvera principalement de la gestion des ressources humaines, la solution a posteriori pouvant aller jusqu'à des sanctions graves telles que le licenciement ou des poursuites judiciaires. Ici encore, de nombreux champs de recherche se présentent à nous. Comment constituer un dossier de preuves, comment communiquer en interne la motivation et la nature des sanctions prises, comment rétablir la confiance, quelles mesures de formation et quelles améliorations peuvent être apportées au système de protection existant sont autant de questions sur lesquelles la littérature actuelle reste muette. À cet égard, le fait que notre étude ait démontré 
que tous les secteurs sont concernés par la délinquance plaide en faveur d'un élargissement du champ d'investigation des quelques rares études qui se sont concentrées sur les secteurs considérés comme les plus sensibles (commerce de détail ou banque).

\section{Bibliographie}

Calavita, K., H. Pontell et R. Tillman (1997), Big Money Crime : Fraud and Politics in the Savings and Loan Crisis, Berkeley, University of California Press.

ERnst \& Young (2000), Fraud: The Unmanaged Risk. An International Survey of the Effect of Fraud on Business, Fraud Investigation Group.

Felson, M. et R.V. Clarke (1997), Business and Crime Prevention, New York, Monsey, Criminal Justice Press.

GILL, M. (1998), «The victimisation of business : indicators of risk and the direction of future research », International Review of Victimology, vol. 6, p. 17-28.

Holt, A. (1993), «Controlling employee theft», CMA Magazine 67, septembre, p. 16-19.

Johnston, V., M. Leitner, J. Shapland et P. Wiles (1994), «Crime business and policing on industrial estates », dans M. Gill (dir.), Crime at Work: Studies in Security and Crime Prevention, Leicester, Perpetuity Press, p. 102-123.

KPMG (1999), Belgium Fraud Survey Report, Forensic Services.

KuratKo, D.F., J.S. Hornsby, D.W. NAFFZIGER et R.M. HodgetTs (2000), «Crime and small business: an exploratory study of cost and prevention issues in U.S. firms », Journal of Small Business Management, vol. 38, n 3, p. 1-26.

Punch, M. (1996), Dirty Business : Exploring Corporate Misconduct: Analysis and Cases, Londres, Sage Publications.

U.S. DePartment of Commerce (1995), Costs of Crime Against Business, Washington, D.C., U.S. Government Printing Office.

U.S. Small Business Administration (2000), «Curtailing crime - inside and out», Crime Prevention Series, Washington, D.C., U.S. Government Printing Office.

VAN DIJK, J.J.M. et G.J. TERLOUW (1996), «An international perspective of the business community as victims of fraud and crime», Security Journal, vol. 7, p. 157-167.

VAN DIJK, VAN SOOMEREN \& PARTNERS BV (1990), Bedrijfsleven en criminaliteit: Kengetallen uit de eerste Nederlandse slachtofferenquète onder bedrijven, 1988, Den Haag, Ministerie van Justitie, Directie Criminaliteitspreventie.

Walker, J. (1994), The First Australian Survey of Crimes Against Business, Canberra, Australian Institute of Criminology.

WALKER, J. (1996), «Crime prevention by businesses in Australia », International Journal of Risk, Security and Crime Prevention, vol. 1, $\mathrm{n}^{\circ}$ 4, p. 279-291. 